\title{
Nonlinear Modeling of Tunnel Diode Detectors ${ }^{*}$
}

\author{
D.K. Walker, K.J. Coakley, and J. D. Splett \\ National Institute of Standards and Technology \\ Boulder, CO 80305, U.S.A. \\ dwalker@boulder.nist.gov
}

\begin{abstract}
We investigate the sensitivity and nonlinear properties of a tunnel diode microwave detector as functions of the input power and the load impedance presented at the detector's output. We estimate the systematic error associated with using a linear calibration model to estimate received power and, hence, brightness temperature from the nonlinear voltage-power transfer function of the detector. One estimate is provided by the two-tone method, the other by an empirical regression spline method.
\end{abstract}

Keywords-calibration, microwave radiometer, nonlinearity, radiometer, remote sensing

\section{INTRODUCTION}

Microwave remote sensing radiometers frequently use tunnel diode detectors. Often, nonlinearity in the diode detector is the primary cause for nonlinearity in the radiometer transfer function. Various methods have been used to model and account for the nonlinear detector response, including the twotone method, the constant-ratio method, and the amplitudemodulation method $[1,2]$. The two-tone method, in particular, has been favored because of its potential to provide estimates of relative brightness temperature error with very low variability. However, careful implementation of each method is essential to achieving useful results. Also, the basic assumption that the detector nonlinearity is accurately modeled by a quadratic function may not always hold. An independent means of verifying the methods and assumptions is needed.

In principle, a measurement of the voltage output versus power input of the detector is preferable, since no assumptions are made as to the form of the detector nonlinearity. The measurement then closely mimics how the detector is used in the radiometer. This would facilitate empirical modeling of the detector transfer function, which can then be used to determine a suitable operating range or nonlinearity correction, or both.

While the voltage output can be measured with very low uncertainty, measuring the power incident on the detector is difficult: the linearity of a typical commercial power meter is often no better than $3 \%$ over its operating range. For example, in order for the brightness temperature error due to receiver nonlinearity to be less than $0.05 \mathrm{~K}$, as specified for many of the channels on the Advanced Technology Microwave Sounder (ATMS) on NPOESS, the detector must be linear to better than $0.02 \%$ for a typical radiometer.

We seek to demonstrate the feasibility of direct measurements of the voltage-power (V-P) transfer function for a tunnel diode detector for the dual purposes of providing a means for comparison to the two-tone method, as well as for

\footnotetext{
${ }^{*}$ U.S. government work; not protected by U.S. copyright.
}

developing suitable nonlinear models for fitting the detector response. Careful linearization of the power meter allows us to perform accurate V-P measurements. Results of the two characterization methods are then presented and compared.

\section{Detector Power-Voltage CHARACTERIZATION}

\section{A. Power meter linearization - Setup}

Fig. 1 shows the experimental setup for characterizing the microwave power meter. Two commercial diode noise sources are combined, amplified, and passed through a band pass filter of $5 \%$ bandwidth centered at $1 \mathrm{GHz}$. All measurements were performed in a laboratory environment controlled to within $\pm 0.5^{\circ} \mathrm{C}$. The amplifiers were temperature controlled to within $\pm 0.01{ }^{\circ} \mathrm{C}$ by use of water jackets. The procedure follows the common principle of a two-position attenuator method [3] except for the substitution of a second source and a power combiner in place of the attenuator. Switching one source on and off creates a nearly constant power ratio over a wide range of step attenuator settings with no random error due to connection repeatability or impedance mismatch changes. The power meter employs a diode sensor. Note that we do not require an accurate absolute power calibration, but we do need the response to be very linear for detector V-P characterization.

\section{B. Power meter linearization procedure}

In a power meter linearization experiment, we measure many pairs of powers $\left(\boldsymbol{P}_{L}, \boldsymbol{P}_{U}\right)$. For each pair, we assume that the ratio of the unobserved true powers is well determined, but the absolute value of either true power is not. Due to the additive bias and nonlinearities in the power meter, the observed ratio for the $i$ th pair $\boldsymbol{P}_{U}(i) / \boldsymbol{P}_{L}(i)$ differs, on average, from the true power ratio $\boldsymbol{r}_{\text {true }}$. To demonstrate this, we fit a linear model $\boldsymbol{P}_{U}=\boldsymbol{\alpha} \boldsymbol{P}_{L}+\boldsymbol{\varepsilon}$, where $\boldsymbol{\alpha}$ is the model parameter and $\boldsymbol{\varepsilon}$ is the prediction error, to the power pair data. Since the fractional residuals $\left(\boldsymbol{P}_{U}-\hat{\alpha} \boldsymbol{P}_{L}\right) / \boldsymbol{P}_{U}$ clearly have structure (Fig. 2 ), the power meter is nonlinear. We seek a nonlinear correction model to predict the true power in terms of the observed powers.

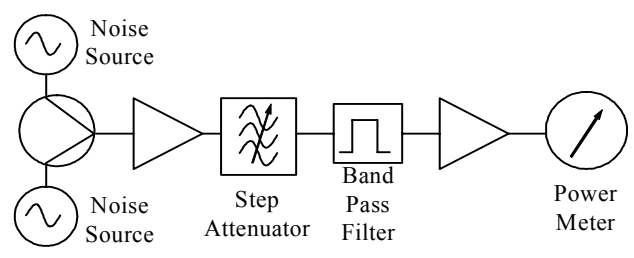

Fig. 1 Power meter calibration setup. 


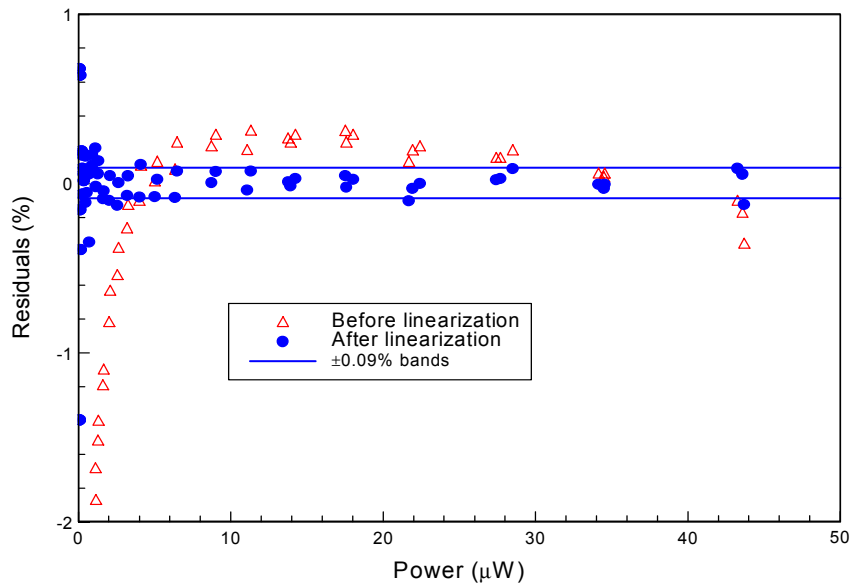

Fig. 2 Residuals before and after power meter linearization.

Based on measured power $P_{m}$ (either $P_{L}$ or $P_{U}$ ) our predicted value of the true power $\hat{P}$ is

$$
\hat{P}=f\left(P_{m}\right)=S P_{0}\left(\frac{P_{m}}{P_{0}}-\Delta\right)^{g\left(\frac{P_{m}}{P_{0}}-\Delta\right)},
$$

where

$$
g(x)=a_{0}+\sum_{k=1}^{5} a_{k} x^{k}
$$

In (1), the model parameters to be determined form a sevendimensional vector $\left(\Delta, a_{0}, a_{1}, a_{2}, a_{3}, a_{4}, a_{5}\right), P_{0}$ is a reference power (the maximum power observed is approximately $44 \mu \mathrm{W}$ ), and $S$ is a scale factor. We set $P_{0}$ to the maximum measured power in the calibration data set. We model the additive bias as the product $\Delta P_{0}$, where $\Delta$ is a dimensionless model parameter to be determined. Based on prior knowledge of the noise temperature of the two noise sources and the properties of the power combiner, we set $r_{\text {true }}$ $=1.73$. We determined the model parameters by minimizing the mean square prediction error as follows:

$$
M S P E=\sum_{i}\left(\hat{r}(i)-r_{\text {true }}\right)^{2},
$$

where the predicted ratio is

$$
\hat{r}(i)=\frac{f\left(P_{U}(i)\right)}{f\left(P_{L}(i)\right)} .
$$

The predicted ratio $\hat{r}$ does not depend on the scale factor $S$. We determined $S$ by requiring that the maximum predicted power $f\left(P_{0}\right)=P_{0}(1-\Delta)$. This implies that $S=(1-\Delta)^{1-g(1-\Delta)}$. The estimated values of the model parameters of (4) are $\left(\Delta=0.000912254, a_{0}=0.98756\right.$, $a_{1}=0.067652, \quad a_{2}=-0.31618, \quad a_{3}=0.93176$, $\left.a_{4}=-1.28642, a_{5}=0.66817\right)$. In Fig. 2 , we plot fractional residuals about a linear regression model of $f\left(P_{U}\right)$ on $f\left(P_{L}\right)$ (no intercept). The RMS deviation of the residuals for powers above $2 \mu \mathrm{W}$ (54 observations out of the 60-point data set) is $0.09 \%$ (shown as bands in the figure). To understand the magnitude of nonlinearity in the data, we plot a correction factor $C(P)=\frac{f(P)}{P(1-\Delta)}$ in Fig. 3 .

Our results should be interpreted as a proof-of-principle effort to establish the feasibility of a power linearization method. Because of the limited amount of data collected for this study, we cannot present a full uncertainty analysis of the corrected power measurements. In future studies, we plan to quantify sources of variability including random sampling errors, instrumental drift due to environmental effects, and uncertainty in the prior knowledge of $r_{\text {true }}$.

\section{Detector voltage-power characterization}

Figure 4 shows the experimental setup for measuring the detector voltage output as a function of power input. The detector is a commercially-packaged tunnel diode with an operating range of $0.5-2.0 \mathrm{GHz}$ and a nominal output impedance of $125 \Omega$. The detector was temperature-controlled using a water jacket (separate from the amplifiers) so that its operating temperature could be varied.

In Fig. 5, we plot detector sensitivity versus ratios of voltage and corrected power for three values of the load impedance $Z_{L}$, all at a detector temperature of $20^{\circ} \mathrm{C}$. Fig. 6 shows similar curves for three different detector temperatures with $Z_{L}=125 \Omega$. The voltage is corrected for an additive bias by requiring that the predicted value of voltage from a linear model be zero when power is zero.

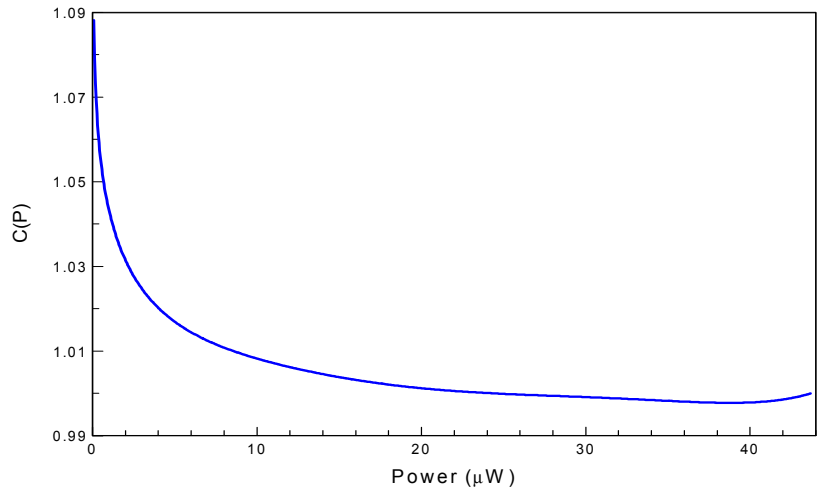

Fig. 3. Power meter correction factor.

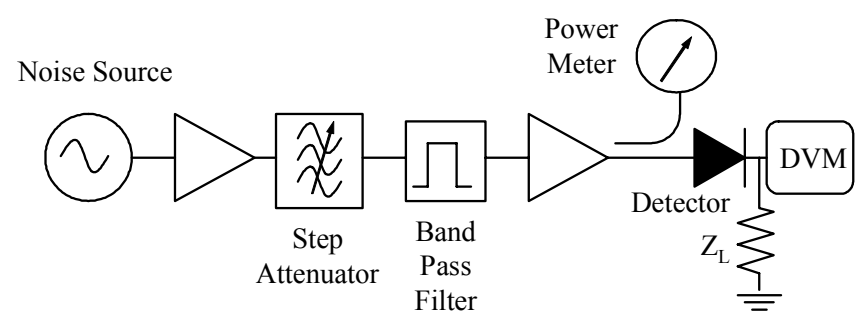

Fig. 4 Detector voltage-power measurement setup. 


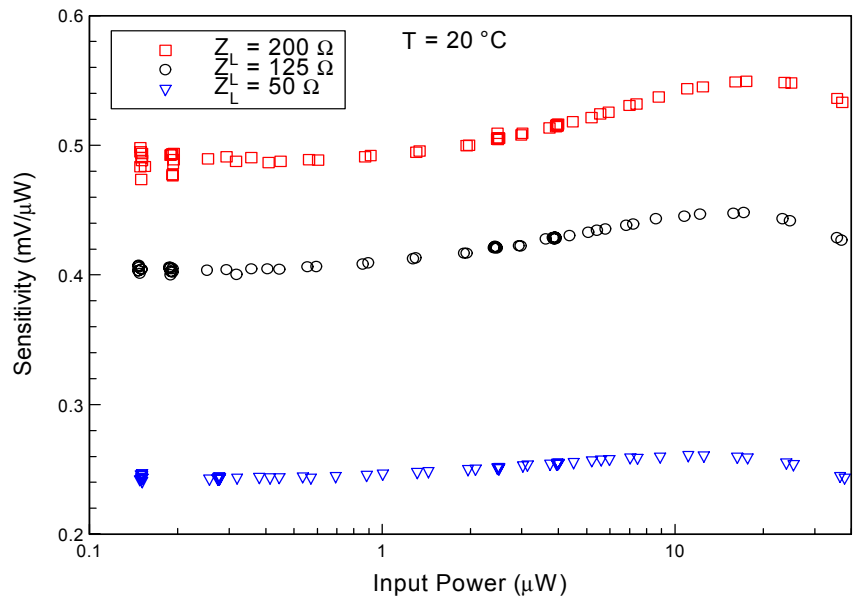

Fig. 5 Detector sensitivity at $\mathrm{T}=20^{\circ} \mathrm{C}$.

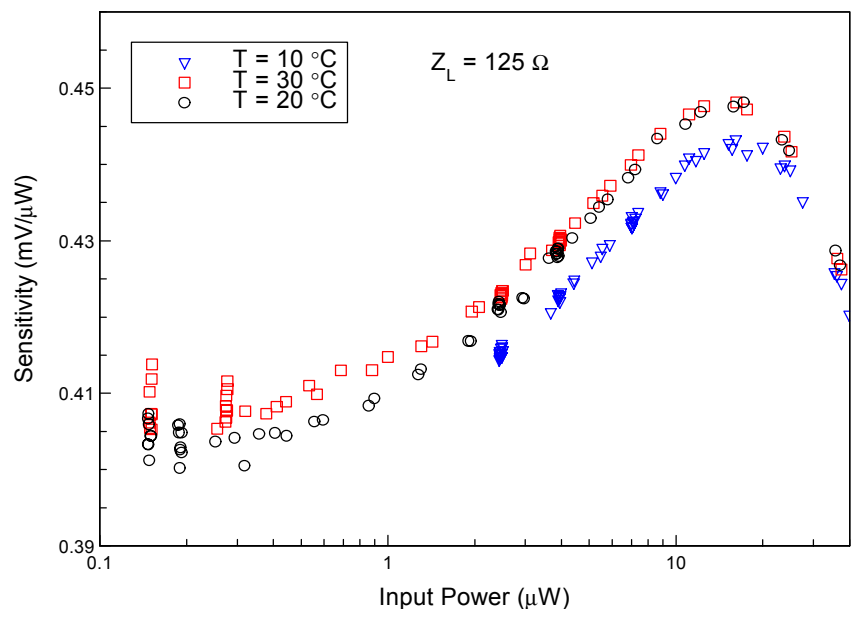

Fig. 6 Detector sensitivity as a function of power for $Z_{L}=125 \Omega$.

The flat response at very low input power is typical of tunnel diode detectors. The "super-linear" behavior (i.e., where the sensitivity increases with input power) has been described for the case of Schottky detector diodes [4]. This complicated behavior is not predicted by the polynomial model in [1]. Fig. 5 indicates that the detector can be modeled as a voltage source with a series output resistance of about $100 \Omega$. The sensitivity has a flatter response as $Z_{L}$ decreases: the ratios of the peak sensitivity to the low-power sensitivity for the three curves are, respectively, $1.13\left(\mathrm{Z}_{\mathrm{L}}=200 \Omega\right), 1.11\left(\mathrm{Z}_{\mathrm{L}}=125 \Omega\right)$, and 1.07 $\left(Z_{\mathrm{L}}=50 \Omega\right)$. Although there is a weak dependency of sensitivity with temperature (Fig. 6), the shape of the response is similar. These sensitivity results are in qualitative agreement with independent measurements (using a different technique) of a similar detector performed at the NASA Jet Propulsion Laboratories [5].

We modeled the measured voltage-power curves as a cubic $\mathrm{B}$-spline where the number of degrees of freedom of the spline is selected by cross-validation [6]. The abscissa is the corrected power and the ordinate is the measured voltage. Based on a measurement of voltage, a linear interpolation model to predict power $P$ is

$$
\hat{P}=P_{1}+\frac{V(P)-V\left(P_{1}\right)}{V\left(P_{2}\right)-V\left(P_{1}\right)}\left(P_{2}-P_{1}\right) .
$$

In Eq. (5), $P_{1}, P_{2}, V\left(P_{1}\right), V\left(P_{2}\right)$, and $V(P)$ are observed, but $P$ is not.

\section{Two-Tone Method}

Fig. 7 shows the experimental setup for two-tone testing. The two-tone measurement procedure was similar to that described in [1], with the notable exception that the load impedance at the detector output was carefully controlled. The input impedance of the spectrum analyzer is about $39 \Omega$. This may not be the optimal or even the typical load impedance for practical applications. The isolation between the two sources was verified by looking at intermodulation products on the spectrum analyzer with no detector. The highest intermodulation product was $56 \mathrm{~dB}$ below the source power level. Accordingly, the predicted resolution for the radiometric brightness temperature error for a receiver with a noise figure $F$ of $4 \mathrm{~dB}$ and an input temperature range of $300 \mathrm{~K}$ is about 3 $\mathrm{mK}$, assuming the polynomial model is perfectly applicable for this detector.

We measured the base-band harmonic power as a function of input power, load impedance $Z_{\mathrm{L}}$, and detector temperature. Fig. 8 shows an example of the harmonic power vs. input power for $Z_{L}=100 \Omega$. The deviation in the curves from constant slope and the dramatic increase in the power of higher-order harmonics at higher input power indicates that the detector is not accurately modeled by a quadratic polynomial.

We also tested the detector at different operating temperatures and found no discernable changes in the harmonic response. This is not to say that the sensitivity is unchanged; merely that the nonlinearity does not vary significantly. We also measured the harmonic output of the IF amplifiers used in the V-P measurements (Fig. 4). At the highest power level used in this experiment, the amplifier harmonic power levels were at least $30 \mathrm{~dB}$ below the detector harmonic power levels.

\section{NUMERICAL EXAMPLE}

We estimated the systematic error associated with a linear interpolation method for predicting power (or brightness temperature) in terms of measured voltage based on calibration data $\left(P_{1}, V\left(P_{1}\right)\right)$ and $\left(P_{2}, V\left(P_{2}\right)\right)$. We assumed that temperature and power are linearly related. In our example we considered a radiometer with a low-noise receiver $\left(F=1.8 \mathrm{~dB} ; T_{N}=150 \mathrm{~K}\right)$ and an operating range between the calibration temperatures of $0 \mathrm{~K}$ and $300 \mathrm{~K}$. The detector was set to operate between $8 \mu \mathrm{W}$ $(-21 \mathrm{dBm})$ and $24 \mu \mathrm{W}(-16.2 \mathrm{dBm})$ input power with an output load impedance $Z_{\mathrm{L}}=50 \Omega$.

In Fig. 9 we plot the estimated systematic error determined from Eq. (5) using our spline model prediction for $V(P)$. In our study, we assumed that all powers and voltages were measured without random error. Hence, we quantify only systematic error. For the same example, the two-tone model predicts a maximum systematic error of $5.0 \mathrm{~K}$. 


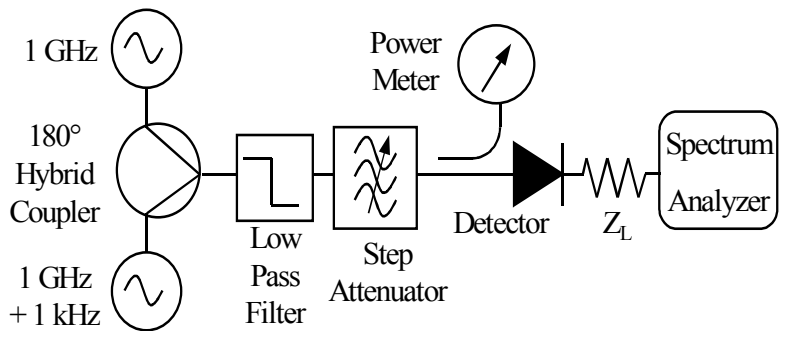

Fig. 7 Two-tone measurement setup.

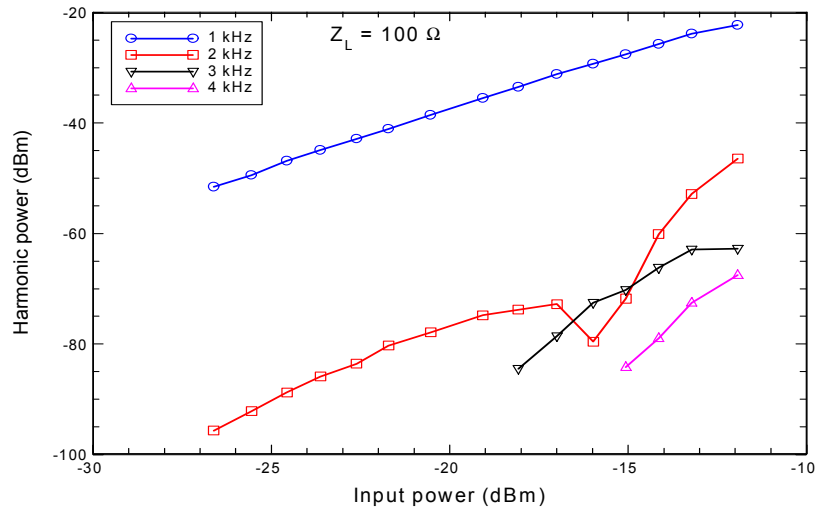

Fig. 8 Detector harmonic output for $Z_{L}=100 \Omega$.

The variability in our systematic error is due solely to uncertainty of the true V-P curve that we estimated as a cubic B-spline. We used a nonparametric bootstrap method [7] to resample the V-P curve. For each resampled curve, i.e., each bootstrap replication of the observed data, we refit the spline model and reestimated the linear interpolation error using the same procedure employed for the observed data. We plot the estimate from the observed V-P curve and $\pm 1 \sigma$ bands in Fig 9. In this approach, the corrected power (the abscissa) is treated as deterministic. In the future, we plan to collect more data to validate our spline model estimate of the systematic uncertainty due to linear interpolation and to quantify random variability associated with the interpolation scheme.

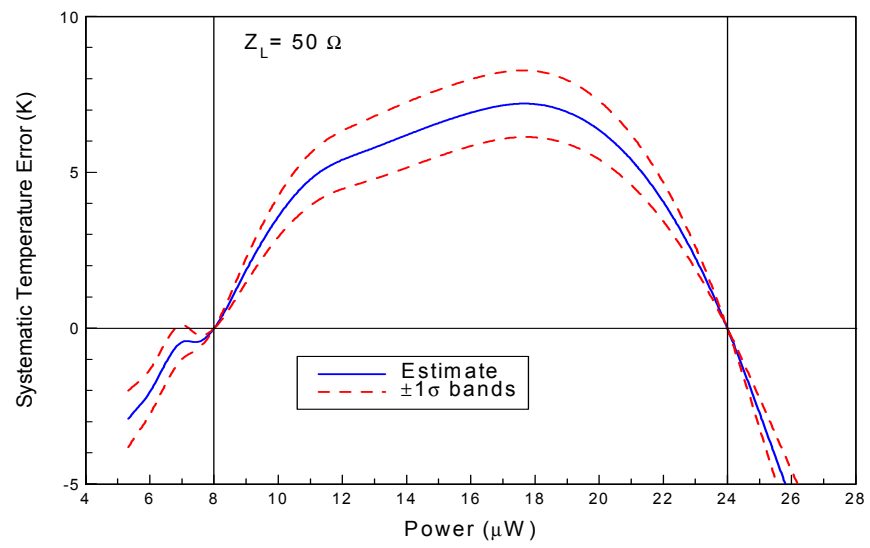

Fig. 9 Estimate of systematic error associated with linear interpolation method based on spline fit model for V-P curve.

\section{SUMMARY}

The detector's sensitivity and nonlinear properties are a function not only of the input RF power, but also of the load impedance presented at the detector's output. Selection of the load impedance is guided by the goal of minimizing the nonlinearity of the detector while preserving adequate sensitivity. Lower load impedance improved the uncorrected nonlinear detector response at the expense of output signal voltage. The detector's temperature had only a small effect on either sensitivity or nonlinear characteristics.

The assumption that the transfer function is well approximated by a global polynomial model, as used in the two-tone method, is only valid in special cases (e.g., low output load impedance at low input power levels). The load impedance should be controlled when performing any detector characterization measurements because of these effects.

V-P characterization offers the opportunity to develop accurate global models for the detector transfer function along with uncertainty estimates. We demonstrated the use of a global regression spline model. In contrast to polynomial models, the validity of a regression spline model is not restricted to certain operating conditions. In conclusion, the systematic uncertainty of the brightness temperature, as inferred from a calibration experiment, depends on the operating conditions, the test method used for evaluating the detector, and the particular prediction model for the nonlinear response of the tunnel diode detector.

\section{ACKNOWLEDGMENT}

We thank Jeff Piepmeier of the NASA Goddard Space Flight Center for loan of the detector and IF amplifier.

\section{REFERENCES}

[1] V.S. Reinhardt, Y.C. Shih, P.A. Toth, and A. L. Berman, "Nethods for Measuring the Power Linearity of Microwave Detectors for Radiometric Applications," IEEE Trans. Microwave Theory Tech., vol. 43, pp. 715 720, April 1995.

[2] T. Närhi, "Nonlinearity characterisation of microwave detectors for radiometer applications," Electron. Lett., vol. 32, pp. 224- 225, Feb. 1996.

[3] C.A. Hoer, K.C. Roe, and C.M. Allred, "Measuring and minimizing diode detector nonlinearity," IEEE Trans. Instr. Meas., vol. IM-25, pp. $324-329$, Dec. 1976.

[4] R.G. Harrison, "Full Nonlinear Analysis of Detector Circuits Using RitzGalerkin Theory," IEEE MTT-S Digest, 1992.

[5] A. Tanner, NASA Jet Propulsion Laboratories, unpublished.

[6] T.J. Hastie and R.J. Tibshirani, Generalized Additive Models. Chapman and Hall, London. 1990.

[7] B. Efron and R.J. Tibshirani, An Introduction to the Bootstrap, Chapman and Hall, 1993. 